\title{
Possible Cellular Mechanism for Cerebral Vasospasm after Experimental Subarachnoid Hemorrhage in the Dog
}

\author{
David R. Harder, ${ }^{\star *}$ Paul Dembach, ${ }^{* \neq}$ and Alan Waters \\ ${ }^{*}$ Departments of Neurology, Neurosurgery, and Physiology, Medical College of Wisconsin, Milwaukee, Wisconsin 53295; ${ }^{\ddagger}$ Veterans \\ Administration Medical Center, Milwaukee, Wisconsin 53295; and ${ }^{\S}$ Department of Neurosurgery, \\ Adenbrook's Hospital, Cambridge, United Kingdom
}

\begin{abstract}
This study was undertaken to examine some of the cellular ionic mechanisms responsible for the cerebral vasospasm that occurs as a consequence of subarachnoid hemorrhage (SAH). After cisternal injection of autologous blood we documented spasm of the basilar artery upon angiography from 4 to $7 \mathrm{~d}$ postictus in six dogs. When these basilar arteries were isolated we observed marked membrane depolarization and enhanced electrical spike activity compared with controls. The slope of the membrane potential vs $\log \left[K_{0}\right]_{0}$ curve was significantly reduced in arteries exposed to SAH. Further analysis supported the concept that such altered muscle cell properties resulted from reduction in resting $\mathrm{K}^{+}$conductance $\left(\mathrm{g}_{\mathrm{k}}\right)$. Exposure of arteries in vitro to nicorandil $\left(10^{-9}-10^{-7} \mathrm{M}\right)$ (a drug which acts by increasing $g_{k}$ ) hyperpolarized the muscle cells and increased internal diameter. Infusion of nicorandil (3-5 $\mu \mathrm{g} / \mathrm{kg}$ per min) to intact, anesthetized animals reversed, by $50 \%$, the reduction in basilar artery diameter after experimental SAH.
\end{abstract}

\section{Introduction}

Cisternal injection of autologous blood produces spasm of basilar and anterior spinal arteries. This model has been used successfully in cats and dogs in an effort to understand the mechanism involved in the development and maintenance of cerebral vasospasm after subarachnoid hemorrhage $(\mathrm{SAH})^{1}(1-4)$.

Using this model in cats, we have previously found that in basilar arteries exposed to subarchnoid blood there is significant muscle cell membrane depolarization. This reduction in $\mathrm{K}^{+}$conductance $\left(\mathrm{g}_{\mathrm{k}}\right)$ was long lasting as documented by the fact that, when compared with controls, isolated basilar arteries removed from cats 2 and $7 \mathrm{~d}$ post-SAH remained in a

Address reprints and correspondence to Dr. Harder, VA Medical Center, Research Service 151, 5000 W. National Ave., Milwaukee, WI 53295.

Received for publication 9 June 1986 and in revised form 5 February 1987

1. Abbreviations used in this paper: $\mathrm{Em}$, membrane potential; $\mathrm{g}_{\mathrm{k}}, \mathrm{K}^{+}$ conductance; $\mathrm{g}_{\mathrm{Na}}, \mathrm{Na}^{+}$conductance; $\left[\mathrm{K}^{+}\right]$, extracellular $\mathrm{K}^{+} ; \mathrm{P}_{\mathrm{K}}$, potassium permeability; $\mathbf{P}_{\mathrm{Na}}$, sodium permeability; $\mathbf{P}_{\mathrm{Na}} / \mathbf{P}_{\mathbf{K}}$ ratio, ratio of $\mathbf{P}_{\mathrm{Na}}$ and $P_{K} ; r_{i n}$, input resistance; $S A H$, subarachnoid hemorrhage; $\Delta V / I_{0}$, voltage vs. applied current.

J. Clin. Invest.

(c) The American Society for Clinical Investigation, Inc.

0021-9738/87/09/0875/06 \$2.00

Volume 80, September 1987, 875-880 depolarized state for up to $6 \mathrm{~h}(1,5)$. In the present study we set out to determine if there were alterations in the muscle cell membrane of basilar arteries of dogs after double injection of autologous blood, namely alteration in $\mathbf{g}_{\mathbf{k}}$, and if the manifestations of this phenomenon (i.e., membrane depolarization, action potential generation, and an enhanced level of tone) were reversed by a drug that specifically increases $\mathbf{g}_{\mathbf{k}}$ in excitable tissues, namely nicorandil [ $N$-(2-hydroxethyl) nicotinomide] (6-8).

We found that in basilar arteries from double-injected dogs 4-7 dpostictus, the muscle cell membrane was markedly depolarized compared with control, and exhibited spontaneous action potential generation not found in controls. Further electrophysiological analysis produced data compatible with the hypothesis that, in dog basilar arteries isolated from the brain and studied in an organ bath 4-7 d post-SAH, there is a lower muscle membrane $\mathrm{g}_{\mathrm{k}}$ compared with sham-injected controls. Perfusion of the vessels with nicorandil repolarized the muscle cell membrane, abolished action potential generation, and partially reversed cerebral vasospasm in intact animals, further supporting the hypothesis that $\mathrm{SAH}$ reduces $\mathrm{g}_{\mathrm{k}}$ in cerebral arterial muscle.

The active state of arterial muscle is, to a large extent, regulated by $g_{k}(5)$. Significant reduction in $g_{k}$ markedly activates cerebral arterial muscle and increases the sensitivity to vasoactive agents. It is possible that the vasospasm observed after SAH in dogs is mediated by the lowering of $\mathbf{g}_{k}$. If that is the case, then an agent that acts specifically to increase $g_{k}$, namely nicorandil, may provide useful therapy for the treatment of cerebral vasospasm after SAH.

\section{Methods}

Studies were done using tissue from mongrel dogs $12-18 \mathrm{~kg}$ in weight. The animals were anesthetized with intravenous sodium-pentobarbital $(25 \mathrm{mg} / \mathrm{kg}$ ), and prepped and draped under sterile conditions for cisternal injection of autologous blood obtained from a femoral arterial catheter. Cisternal puncture was accomplished using a 20-g spinal needle. $3 \mathrm{ml}$ of cerebral spinal fluid was removed and subsequently replaced with an equal amount of arterial blood. Animals were then allowed to recover. $2 \mathrm{~d}$ later the dogs were again anesthetized and another 3-4 $\mathrm{ml}$ of arterial blood (from the femoral artery) was injected into the cisternal space. Animals were monitored continually during the procedures and four to six times daily thereafter by animal facility personnel to assure maximum comfort and humane care.

From 4 to $7 \mathrm{~d}$ after the second injection the dogs were again anesthetized and basilar artery spasm documented via angiography. Angiograms were performed by threading a 5 French catheter into the left vertebral artery percutaneously via the right femoral artery. Catheter placement was at the C-3 to C-4 level and was confirmed by fluoroscopy. The circulation was visualized via rapid bolus $(5 \mathrm{ml})$ injection of Renografin-60. All $x$ rays were taken at a constant distance, incidence angle, and time after dye injection. The data presented below is from 
six dogs in which we documented a $>50 \%$ reduction in basilar artery diameter. Fig. 1 documents the degree of spasm of the basilar artery in a dog $5 \mathrm{~d}$ postictus. Control angiograms were obtained in all dogs just before the first cisternal injection of autologous blood. Measurements of diameter were obtained by projecting the angiographic image, via a photographic enlarger, to a size of $3.6 \times$ the original.

After documentation of spasm the brain was quickly removed. Basilar arteries were dissected free of arachnoid and placed in cold $\left(4^{\circ} \mathrm{C}\right) \mathrm{Kreb}$ 's solution $\left(\mathrm{HCO}_{3}{ }^{-}\right.$buffered). Within an hour of removal, isolated segments $(\sim 1 \mathrm{~cm}$ in length) were threaded onto pipettes at either end and tied in place with $22-\mu \mathrm{m}$ silk suture. All side branches were tied off with similar suture material. One pipette was connected to a pressure reservoir in series with an appropriate transducer to allow manipulation and precise monitoring of applied transmural pressure. The transmural pressure was maintained at the mean arterial pressure of the animal $(\sim 80-100 \mathrm{mmHg})$. Internal diameter of the basilar artery segments was monitored via a high resolution binocular microscope (Zeiss) that had a trinocular tube connected to a video camera, the image of which was displayed on a Colorado video image splitter, which measured diameter to the nearest micron. We studied arteries from six animals 4-7 d post-SAH in which we documented marked reduction in diameter angiographically. Nicorandil was perfused through the vessels at the mean arterial pressure of that particular animal (refer to above). All arteries in vitro were perfused and suffused in a muscle myograph with a physiological salt solution containing (in millimolars): $141, \mathrm{Na}^{+} ; 4.7, \mathrm{~K}^{+} ; 2.5, \mathrm{Ca}^{2+} ; 0.72, \mathrm{Mg}^{2+} ; 124, \mathrm{Cl}^{-} ; 1.7$, $\mathrm{H}_{2} \mathrm{PO}_{4} ; 24, \mathrm{HCO}_{3}{ }^{-}$; and 11.0 , glucose. Solutions were aerated with $94 \% \mathrm{O}_{2} / 6 \mathrm{CO}_{2}$ and yielded a pH of 7.37-7.40 and $\mathrm{PCO}_{2}$ of 37-40 torr. Solution gases were monitored via periodic sampling using a Radiometer gas analyzer. Temperature was maintained at $37^{\circ} \mathrm{C}$ via a water jacket.

Electrophysiological analysis of intracellular events from muscle of dog basilar arteries from animals 4-7 d post-SAH $(n=6)$ and shaminjected controls $(n=7)$ was obtained using glass microelectrodes filled with $3 \mathrm{M} \mathrm{KCl}$ and having tip impedances of 50-80 M 2 . Details of electrophysiological analysis have been published previously (9). Membrane potential $\left(\mathrm{E}_{\mathrm{m}}\right)$ vs $\log$ extracellular $\mathrm{K}^{+}\left(\left[\mathrm{K}_{0}\right)\right.$ curves were obtained in arteries from animals exposed to $\mathrm{SAH}$ and compared with arteries obtained from sham-operated (i.e., removal and subsequent reinjection of cisternal cerebral spinal fluid) controls in an effort to understand some of the ionic mechanisms of the observed muscle cell depolarization in arteries exposed to subarachnoid blood. $[\mathrm{K}]_{0}$ was elevated in doubling increments $\left(2,6,10,11\right.$, etc). Extracellular $\mathrm{Na}^{+}$ was manipulated to keep the sum of extracellular $\mathrm{Na}^{+}$plus $[\mathrm{K}]_{0}$ constant to maintain osmolarity constant. When $[\mathrm{K}]_{0}$ was elevated beyond $8 \mathrm{mM}$ the bathing solutions were switched to ones containing normal $\mathrm{K}^{+}$for 20-30 min before each consecutive doubling step to avoid $\mathrm{K}^{+}$ accumulation by the muscle cells. Extrapolation of the steepest portion of the curve, when measured in the presence of nicorandil, to zero membrane potential estimated an intracellular $[\mathrm{K}]_{\mathrm{i}}$ of $\sim 180 \mathrm{mM}$, a value not greatly higher than measured chemically (10). In cells in which the $E_{m}$ is regulated primarily by $g_{k}$ and the transmembrane $\mathrm{K}^{+}$ gradient, the membrane depolarizes with a slope between 10 and 100 $\mathrm{mM}[\mathrm{K}]_{0}$ of $61 \mathrm{mV}$ at $37^{\circ} \mathrm{C} \mathrm{(5),} \mathrm{such} \mathrm{as} \mathrm{is} \mathrm{the} \mathrm{case} \mathrm{with} \mathrm{skeletal} \mathrm{and}$ myocardial muscle. Deviation of the slope to one $<61 \mathrm{mV}$ per decade may result from a significant contribution of ions other than $\mathrm{K}^{+}$to maintenance of the $E_{m}$, most likely $\mathrm{Na}^{+}$. Thus, the slope of the $E_{m} v s$. $\log [\mathrm{K}]_{0}$ curve is determined by the ratio of sodium permeability $\left(\mathrm{P}_{\mathrm{Na}}\right)$ to potassium permeability $\left(\mathrm{P}_{\mathrm{K}}\right)\left(\mathrm{P}_{\mathrm{K}} / \mathrm{P}_{\mathrm{Na}}\right.$ ratio) and can be affected by an increase in $P_{\mathrm{Na}}$ or decrease in $P_{K}$. If $S A H$ reduces the slope of the $E_{m}$ vs. $\log [\mathrm{K}]_{0}$ curve, than it does so by increasing muscle membrane $\mathrm{Na}^{+}$ conductance $\left(\mathrm{g}_{\mathrm{Na}}\right)$ (analogous to permeability) or reducing $\mathrm{g}_{\mathrm{k}}$. If $\mathrm{g}_{\mathrm{Na}}$ is increased, then membrane resistance would decrease (conductance is the reciprocal of resistance), and if $g_{k}$ is reduced, membrane resistance would increase. Chloride appears to be passively distributed in many arterial muscle preparations (10) and therefore does not contribute to maintenance of the steady state $E_{m}$.

To help determine if the reduced slope of the $E_{m} v s . \log [K]_{0}$ curve in muscle cells from arteries exposed to subarachnoid blood is due to changes in $\mathbf{g}_{\mathrm{Na}}$ or $\mathrm{g}_{k}$, we measured input resistance $\left(r_{\text {in }}\right)$ of the cells by passing small square wave current pulses through the microelectrodes while inside a cell and measuring associated voltage changes. $r_{\text {in }}$ was estimated by use of a bridge technique. There is some controversy regarding the use of a point source of current injection for measuring $r_{\text {in }}$; however, pressurized arteries as those studied here do not lend themselves to other methods (i.e., partitioned chamber methods), and we are using this technique only as an adjunct to the $E_{m} v s . \log [K]_{0}$ curves and are, thus, looking at trends to assess the relative contributions of $P_{N a} / P_{K}$ in reducing the slope of the $E_{m} v s . \log [K]_{0}$ curve. This technique involves injecting various intensities of current from 1 to 3 $\mathrm{nA}$, and from the concomitant changes in voltage, and constructing a steady state voltage vs. applied current $\left(\Delta V / I_{0}\right)$ curve, the methods of which have been described in detail elsewhere (12). Briefly, current was injected by means of a bridge circuit within the amplifier. The bridge circuit was balanced before microelectrode impalement of a cell. Upon impalement of a cell, rectangular current pulses $\left(I_{0}\right)$ of various intensity were applied through the microelectrode and associated $\Delta V / I_{0}$ curves through the origin constructed. Current was injected in an increasing sequence 1-3 $\mathrm{nA}$ in the hyperpolarizing direction. The current pulses were $30 \mathrm{~ms}$ in duration and total voltage displacement reached saturation. Because one does not know if the bridge circuit is in complete balance while the microelectrode is in the cell, the bridge technique can only be used to compare changes in the same cell (same impalement) before and after manipulation. Thus, $\Delta \mathrm{V} / \mathrm{I}_{0}$ curves were constructed from an impalement before addition of nicorandil, and from the same impalement 2-4 min after addition of the drug. If the impalement was lost upon addition of nicorandil before a second $\Delta V / \mathrm{I}_{0}$ curve could be constructed, the data was discarded. Due to the high resistance electrode used, only small intensity current pulses could be adequately passed (1-3 nA); therefore, rectification was not easily observed. If the bridge was $>1 \mathrm{mV}$ out of balance upon withdrawal of the microelectrode from the cell, that value of $r_{\text {in }}$ was discarded. We constructed $\Delta V / \mathbf{I}_{0}$ curves from the same vessels in which $E_{m} v s . \log [K]_{0}$ curves were obtained. We also measured $r_{\text {in }}$ in arteries exposed to subarachnoid blood before and immediately after nicorandil perfusion to $(a)$ assure ourselves that the difference in slope of the $\Delta V / I_{0}$ curves between SAH and control cerebral arterial muscle was not simply due to differences in junctional resistance between cells which may have occurred as a consequence of $\mathrm{SAH}$; and $(b)$ to help clarify whether or not the observed depolarization in muscle from arteries exposed to subarachnoid blood may be due to changes in $\mathbf{g}_{k}$. Nicorandil exerts its action primarily by increasing $g_{k}$ in excitable tissues (refer to Introduction), and therefore, if in the presence of nicorandil the slope of the $\Delta V / I_{0}$ curve returns to control, it is likely that in arteries exposed to SAH the slope was increased as a consequence of a reduced $g_{k}$. It would be highly unlikely that during the 20-min exposure to nicorandil there would be significant changes in junctional resistances between cells, especially given the low dose $\left(10^{-9} \mathrm{M}\right)$ of nicorandil that we found to be effective.

From 4 to $7 \mathrm{~d}$ postictus five dogs were again anesthetized for analysis of vasospasm and for the effect of nicorandil on the resultant vasospasm after experimental SAH. Angiograms were obtained before and after intravenous administration of nicorandil for $20 \mathrm{~min}$. The dose of nicorandil used in all five dogs studied was between 3-5 $\mu \mathrm{g} / \mathrm{kg}$ per min. We found that these low doses of nicorandil had no significant effect on either blood pressure or heart rate; therefore, any effect we observed upon administration of the drug could not have been due to autoregulation of cerebral blood flow due to reduction in blood pressure. Arterial blood samples were taken at regular intervals throughout the angiograms and analyzed for $\mathbf{P O}_{2}, \mathrm{PCO}_{2}$ and $\mathrm{pH}$ via a Radiometer gas analyzer. All angiographic data were paired, in that the effect of subarachnoid blood and nicorandil, upon angiographic diameter, were compared with the control angiograms in each dog.

\section{Results}

Intracellular $\mathrm{E}_{\mathrm{m}}$ were measured with glass microelectrodes in isolated, cannulated segments of dog basilar artery that had 
been exposed to physiological levels of transmural pressure in the muscle myograph. Basilar arteries that had been exposed to subarachnoid blood, and in which we documented spasm upon angiography (4-7 d postictus) (Fig. 1, $A$ and $B$ ), were significantly depolarized compared with control $(-36 \pm 1.8$ [SE] compared with $-53 \pm 1.12 \mathrm{mV}$ ).

To further characterize the mechanism of the membrane depolarization after $\mathrm{SAH}$ we measured $\mathrm{E}_{\mathrm{m}}$ as a function of $\log [\mathrm{K}]_{0}$. As can be seen in Fig. 2, the slope of this line (between 10 and $100 \mathrm{mM}[\mathrm{K}]_{0}$ ) is $30 \mathrm{mV}$ per decade in arteries exposed to subarachnoid blood. This slope deviates markedly from that observed in control basilar arterial muscle (Fig. 2, dashed line), and from a Nernstian slope of $61 \mathrm{mV}$ per decade for a highly $\mathrm{K}^{+}$-selective membrane. The effect of nicorandil on arteries exposed to subarachnoid blood is to increase the slope of the $E_{m}$ vs. $\log [K]_{0}$ curve to $47 \mathrm{mV}$ per decade (between 10 and $\left.100 \mathrm{mM}[\mathrm{K}]_{0}\right)$. The slope of the $E_{m}$ vs. $\log [K]_{0}$ curve is determined by the $P_{N a} / P_{K}$ ratio. If this slope was reduced because of a reduced $P_{K}$, then a drug that acts specifically to increase $P_{K}$, namely nicorandil, might be expected to return the slope toward control as is seen in Fig. 2.

Steady state $\Delta V / I_{0}$ curves were obtained in six arteries from dogs that had been given experimental SAH 4-7 d earlier, and in which we documented spasm of that artery in vivo. As can be seen in Fig. 3, the slope of the $\Delta V / I_{0}$ curve through zero is significantly $(P<0.001)$ greater than that obtained in five arteries from control animals. Taken at face value such data, together with the $E_{m}$ vs. $\log [K]_{0}$ curves, suggest that $g_{k}$ is reduced in arteries exposed to subarachnoid blood and is responsible for the depolarization and spontaneous electrical activity of these vessels. However, as stated above, when using a bridge technique one can not rule out the possibility of changes in junctional resistance that may change the slope of the $\Delta V / I_{0}$ curve by decreasing the spread of current between contiguous cells. Therefore, $\Delta \mathrm{V} / \mathrm{I}_{0}$ curves were obtained within $20 \mathrm{~min}$ of low dose nicorandil perfusion $\left(10^{-9} \mathrm{M}\right)$. These data showing that nicorandil brings the slope back to control, together with our knowledge that the primary action of this drug is to increase $g_{k}$, supports the concept that arteries exposed to subarachnoid blood are depolarized because of a reduced $\mathbf{g}_{\mathbf{k}}$.

Another property observed in arterial muscle upon reduction of $P_{K}$ and $g_{k}$ is induction of spontaneous electrical spike activity in previously quiescent cells. Fig. 4 demonstrates the existence of spontaneous spike activity in a depolarized basilar arterial muscle cell that had been exposed to subarachnoid blood. As can be seen, perfusion of $10^{-9} \mathrm{M}$ nicorandil in the same artery abolished spontaneous spike activity. Similar regenerative electrical activity was observed in all arteries exposed to subarachnoid blood, but never in sham-injected controls. Under the electrical recordings in Fig. 4 is a histogram depicting the dilatory action of nicorandil in cannulated basilar arteries exposed to physiological levels of transmural pressure $(\sim 100 \mathrm{mmHg})$. There was a mean increase in internal diameter of six hemorrhaged arteries exposed to nicorandil from $336 \pm 20$ (SE) to $442 \pm 16 \mu \mathrm{m}$ (significantly different at $P$ $<0.001$ ). Nicorandil had no effect on non-hemorrhaged basilar arteries at this dose. However, when the dose of nicorandil was increased to $10^{-7} \mathrm{M}$, nicorandil hyperpolarized and dilated non-hemorrhaged cerebral arteries as well. In five cerebral arteries not in spasm and not previously exposed to subarachnoid blood, nicorandil hyperpolarized the muscle cells beginning at $10^{-7} \mathrm{M}$ from $-58 \pm 2.1 \mathrm{mV}$ to $-69 \pm 3.0 \mathrm{mV}$ at
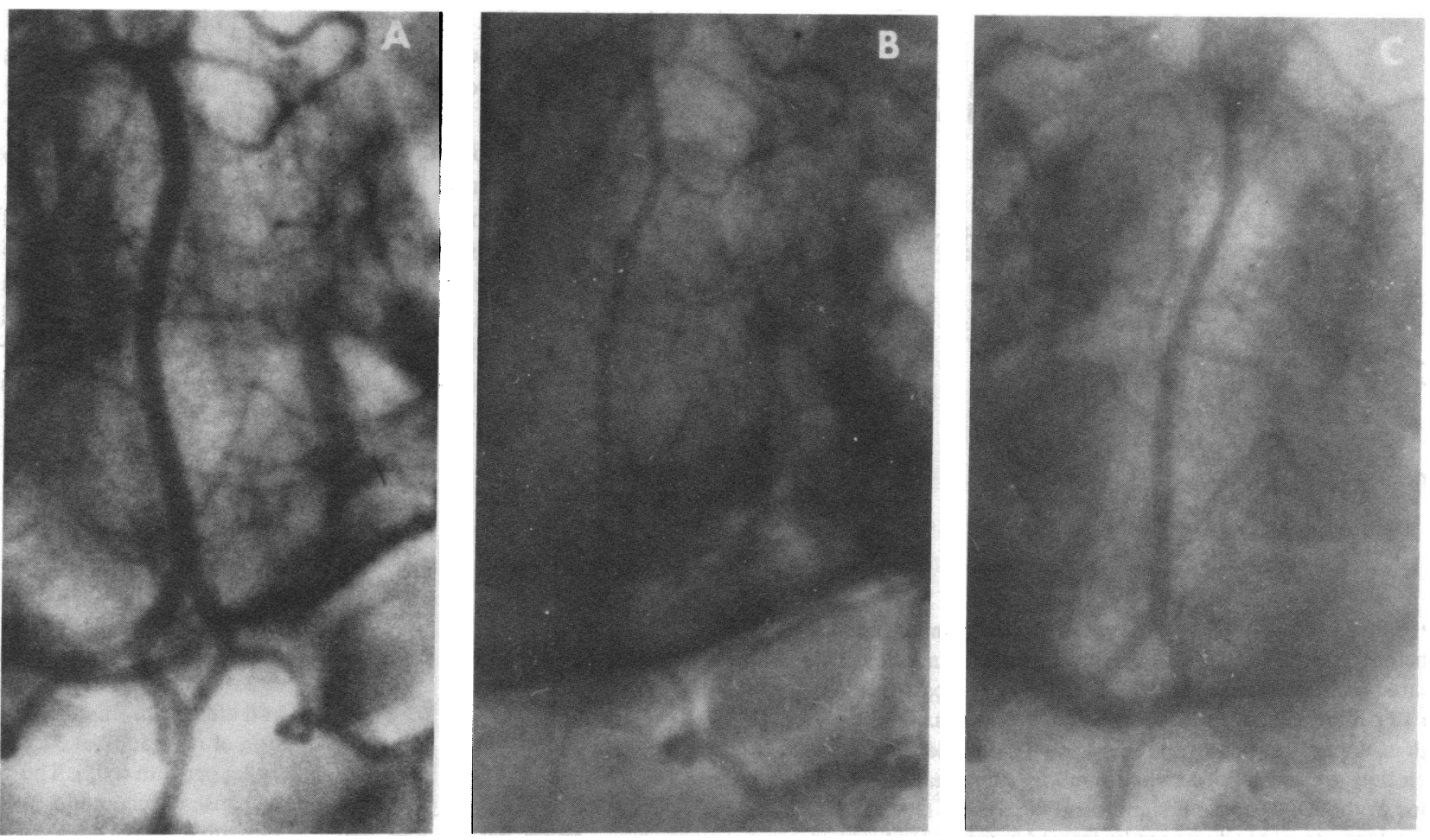

Figure 1. Angiographic visualization of the basilar artery of a dog before $(A)$ and $7 \mathrm{~d}$ after $(B)$ experimental SAH documenting the degree of vasospasm. Frame $C$ depicts an angiogram of the same basilar artery as $B, 20 \mathrm{~min}$ into intravenous infusion of nicorandil at $3 \mu \mathrm{g} / \mathrm{kg}$ per min showing partial reversal of the spasm. 


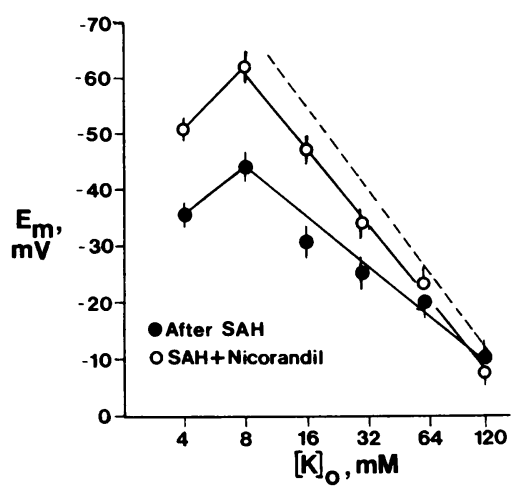

Figure 2. Relationship between $E_{m}$ and external $\mathrm{K}^{+}$concentration $([\mathrm{K}])_{0}$ in basilar arteries removed from dogs 4-7 d postictus (solid circles) and in the same arteries perfused with $10^{-9} \mathrm{M}$ nicorandil (open circles). The dashed line represents the slope of the $E_{m}$ vs. $\log [\mathrm{K}]_{0}$ curve obtained from non-hemorrhaged controls. Lines were fit using linear regression analysis. The lines through each point represent the SE of the mean membrane potential measured from 11 to 19 cells from six different arteries from as many dogs. Note the marked reduction in the slope of the $E_{m} v s . \log$ $[\mathrm{K}]_{0}$ curve measured between 10 and $100 \mathrm{mM}[\mathrm{K}]_{0}$ from cerebral arterial muscle of dogs exposed to SAH when compared with the slope (significantly different at $P<0.05$ ). Note, also, that upon perfusion of the arteries with nicorandil, the slope of this relationship increases toward that observed in control animals.

$10^{-4} \mathrm{M}$, and this was accompanied by significant increases in diameter at a pressure of $100 \mathrm{mmHg}$. Apparently, the membrane depolarization and possible reduced $g_{k}$ in hemorrhaged cerebral arteries increases the sensitivity of agents such as nicorandil.

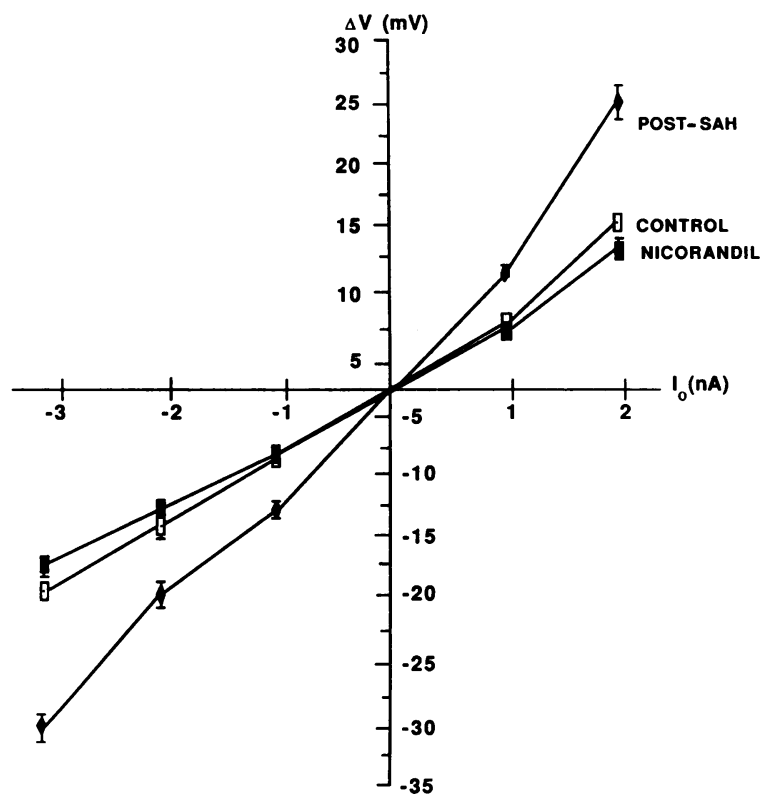

Figure 3. Steady state $\Delta V / I_{0}\left(I_{0}\right)$ curves obtained from dog basilar arteries before (control) and after experimental SAH, and after SAH but treated with nicorandil at $10^{-9} \mathrm{M}$. Each point separates the mean of 8 to 12 cell impalements from six different arteries. Vertical lines through each point represent the SE of the mean; where SE lines are not seen they are overlapped by the symbols. Note that after SAH the slope of the $\Delta V / \mathrm{I}_{0}$ curve is significantly increased $(P<0.01)$, suggesting a decrease in conductance (increased resistance) of one or more ion species. Nicorandil brought the slope back to the control value, demonstrating that the decreased ionic conductance responsible for the increased slope was $g_{k}$.

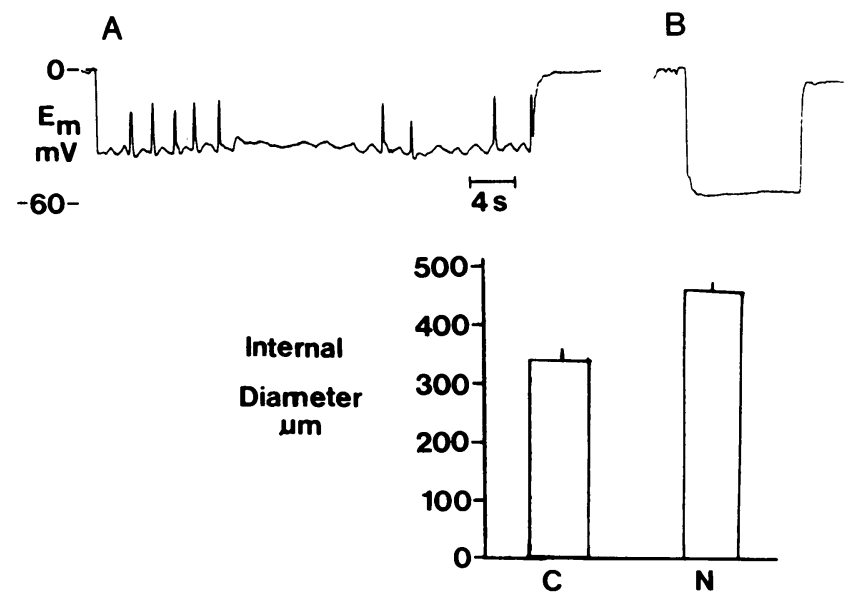

Figure 4. Actual chart recording of intracellular impalements in the same basilar artery (top) removed from a dog $7 \mathrm{~d}$ postexperimental SAH before $(A)$ and after perfusion of the isolated, cannulated arteries with nicorandil $(B)$, showing that nicorandil inhibits spontaneous electrical spike activity. $(A)$ Hemorrhaged artery. $(B)$ Nicorandil $\left(10^{-9} \mathrm{M}\right)$. The histogram below the actual chart records summarizes the internal diameter of six arteries removed from dogs with experimental SAH before (C) and after perfusion of the arteries with nicorandil $\left(10^{-9} \mathrm{M}\right)$. Internal diameter was measured in each artery before and after drug at the same transmural pressure of $\sim 100 \mathrm{mmHg}$.

Again, referring to Fig. 1, we depict an angiogram sequence before SAH $(A$, control), $7 \mathrm{~d}$ postictus $(B)$ depicting the marked vasospasm, and $20 \mathrm{~min}$ after intravenous infusion of nicorandil ( $3 \mu \mathrm{g} / \mathrm{kg}$ per min) showing partial reversal of the spasm $(C)$. Fig. 5 summarizes the data from five such experiments. Note that on the average, nicorandil, at this very low dose, reduces the secondary vasospasm after SAH (4-7 d postictus) by at least $50 \%$. It is conceivable that higher doses would completely reverse the spasm; however, doses beyond $10 \mu \mathrm{g} / \mathrm{kg}$ per min reduce blood pressure, which would complicate the present study due to possible autoregulation (dilation) in response to the fall in blood pressure. $\mathrm{Blood}$ gases $\mathrm{PO}_{2}$ and $\mathrm{PCO}_{2}$ remained within normal limits during the duration of experiments. Within $20 \mathrm{~min}$ of stopping intravenous infusion of nicorandil, the vessels returned to pre-drug diameter, demonstrating that this dose of nicorandil has no long term effect. All angiographic data on each dog is a comparison to the control angiograms taken before injection of subarachnoid blood or infusion of nicorandil. We used the exact anatomical location on the vessels for comparison between control and experimental situations.

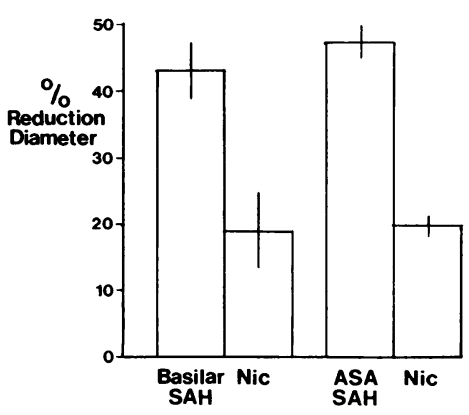

Figure 5. Histogram depicting the percent reduction in basilar and anterior spinal artery (ASA) caliber measured off of angiograms at 3.5 magnification before and after intravenous infusion of nicorandil at 3-5 $\mu \mathrm{g} / \mathrm{kg}$ per min (Nic). The response to nicorandil measured in five dogs postSAH showed a $50 \%$ reduction in both arteries. The line at the top of each bar represents the SE. The response to nicorandil is significantly different at $P<0.05$. 


\section{Discussion}

In the present study we examined arteries from dogs with experimental SAH using the double-injection model. This model is widely used to study the processes involved in the development and maintenance of cerebral vasospasm. Our findings that in arteries exposed to subarachnoid blood and documented to be in spasm there is marked membrane depolarization and action potential generation not found in controls, provides evidence that spasm involves alterations in ionic conductance systems. Further electrophysiological analysis yielded data supporting the concept that the depolarization and spontaneous regenerative electrical activity results from significant reduction in $\mathbf{g}_{\mathbf{k}}$.

A massive sympathetic discharge from adventitial nerves stimulated by subarachnoid blood could result in the observed spasm upon angiography; however, neurotransmitters would be expected to quickly wash out in an organ bath. Furthermore, norepinephrine increases conductance for major ion species, including $\mathrm{K}^{+}$, which would be incompatible with present data. However, such a mechanism in the intact animal could augment the pathological condition set up by muscle cell depolarization, further compounding the problem. This hypothesis is somewhat reinforced in that nicorandil only partially reverses the vasospasm in vivo, suggesting other mechanisms in addition to membrane depolarization. However, in a monkey model of subarachnoid blood-induced cerebrovasospasm, high doses of nicorandil ( $20 \mu \mathrm{g} / \mathrm{kg}$ per min) completely reverse the spasm observed angiographically (preliminary data).

The level of resting tone and degree of activation of arterial muscle is regulated, to a large degree, by the level of resting $\mathbf{g}_{\boldsymbol{k}}$ (5). Reduction of $g_{k}$ by any means markedly increases resting tone and reactivity of arteries (5). Nicorandil has been shown to hyperpolarize arteries from vascular beds other than cerebral, and in several arterial preparations has been shown to markedly increase $\mathrm{K}^{+}$efflux $(3,7,13)$. Furthermore, we have preliminary data (not shown) that nicorandil (beginning at $10^{-8} \mathrm{M}$ ) relaxes the contraction to tetraethylammonium (which acts by decreasing $g_{k}$ ) similar to that observed in guinea pig trachea (4), further supporting the hypothesis that nicorandil acts by increasing $g_{k}$. There are, however, reports that nicorandil increases cyclic-GMP and that part of its mode of action is intracellular, thus requiring a second messenger (14, 15). It appears that whatever action subarachnoid blood has on the muscle cells within these arteries, it does so by exerting a long-lasting effect at the molecular level of the plasma membrane, as evidenced by the change in membrane electrical properties still pesent for many hours after removing these vessels from the brain. We do find that after $6 \mathrm{~h}$ in an organ bath, the cerebral arteries in spasm do start hyperpolarizing toward control levels; however, it is difficult to speculate beyond this observation due to time-dependent deterioration of in vitro preparations.

One important finding of this study is that the perturbation in membrane electrical properties is not permanent even $7 \mathrm{~d}$ after the second injection of autologous blood. The action of nicorandil in this regard is dramatic in that it restores $E_{m}$ back to control, significantly reduces the level of active tone, and abolishes the excitatory electrical activity at a very low dose of $10^{-9} \mathrm{M}$, and does so by luminal administration. Nicorandil is currently in use for treatment of coronary vasospasm (angina pectoris) in humans $(16,17)$, and there is at least one abstract that demonstrates its' vasodilatory action in cerebral vessels (18). The data showing that nicorandil partially reverses established cerebral vasospasm in vivo in this dog model strengthens the hypothesis that one of the mechanisms of cerebral vasospasm after SAH may be the reduction of muscle membrane $g_{k}$ and the subsequent depolarization and excitatory electrical activity. However, other mechanisms such as modulation in the level of cyclic nucleotides may also be involved.

Attempts in defining the substance(s) responsible for vasospasm after SAH have, to date, not been satisfying (19). Similarly, many agents, including $\mathrm{Ca}^{2+}$ channel blockers, have been used in an attempt to reverse the secondary vasospasm after SAH with only moderate success $(11,19,20)$. Our approach was to define the cellular mechanisms of action in an animal model of this disease state as an initial attempt to understand a possible basis for pharmacological intervention.

\section{Acknowledgments}

This study was supported by National Institutes of Health grants 33833 and 31871 and by the Veterans Administration. Dr. Harder is an Established Investigator of the American Heart Association and a Research Career Scientist of the Veterans Administration.

\section{References}

1. Waters, A., and D. R. Harder. 1985. Altered membrane properties of cerebral vascular muscle following subarachnoid hemorrhage in cat: an electrophysiological study. Changes in resting membrane potential $\left(E_{m}\right)$ and effect of the electrogenic pump potential contributing to $\mathrm{E}_{\mathrm{m}}$. Stroke. 16:990-997.

2. Kassel, N. F., T. Sasaki, A. Colohan, and G. Nazure. 1985. Cerebral vasospasm following aneurysmal subarachnoid hemorrhage. Stroke. 16:562-572.

3. Itoh, T., K. Furukawa, M. Kajiwara, K. Kitamura, H. Suzuki, Y. Ito, and H. Kuriyama. 1981. Effects of 2-nicotinamidoethyl nitrate on smooth muscle cells and on adrenergic transmission in the guinea pig and porcine mesenteric arteries. J. Pharmacol. Exp. Ther. 218:260270.

4. Allen, L., R. W. Foster, G. P. Morsan, and R. C. Small. 1986. The relaxant effect of nicorandil in guinea pig isolated trachealis. $B r . J$. Pharmacol. 187:117-127.

5. Harder, D. R., and A. Waters. 1984. Electrical activation of arterial muscle. In International Review of Cytology. G. Bourne and J. Danielli, editors. Academic Press, Orlando, FL. 137-149.

6. Imanishi, S., M. Arita, M. Aomine, and T. Klyosue. 1984. Antiarrhythmic effects of nicorandil on canine cardiac purkinje fibers. $J$. Cardiovasc. Pharmacol. 6:772-779.

7. Furukawa, K., T. Itoh, and M. Kajiwara. 1981. Vasodilatory actions of 2-nicotinamidoethyl nitrate on porcine and guinea-pig coronary arteries. J. Pharmacol. Exp. Ther. 218:248-259.

8. Nakya, H., K. Kanda, Y. Oyama, and T. Sato. 1979. Pharmacokinetics of SG-75 (2-nicotinamidoethyl nitrate) and its hemodynamic effect. Jap. J. Clinical Pharmacol. Ther. 10:116-118.

9. Harder, D. R. 1984. Pressure-dependent membrane depolarization in cat middle cerebral artery. Circ. Res. 55:197-202.

10. Harder, D. R., and N. Sperelakis. 1978. Membrane electrical properties of vascular smooth muscle from the guinea pig superior mesenteric artery. Pfluegers Arch. Eur. J. Physiol. 378:111-119.

11. Kassel, N. F., and C. G. Drake. 1982. Timing of aneurysm surgery. J. Neurosurg. 10:514-519.

12. Harder, D. R. 1982. Membrane electrical activation of arterial smooth muscle. In Vascular Smooth Muscle. M. Crass and C. D. Barnes, editors. Academic Press, Inc., New York. 71-97. 
13. Karashima, T., T. Itoh, and H. Kuriyama. 1982. Effects of 2-nicotinamidoethyl nitrate on smooth muscle cells of the guinea pig mesenteric and portal veins. J. Pharmacol. Exp. Ther. 221:472-480.

14. Holzmann, S. 1983. Cyclic GMP as a possible mediator in coronary arterial relaxation by nicorandil (SG-75). J. Cardiovasc. Pharmacol. 5:364-370.

15. Endo, M., and N. Taira. 1983. Relationship between relaxation and cyclic GMP formation by nicorandil in canine mesenteric artery. Naunyn-Schmiedeberg's Arch. Pharmacol. 322:319-321.

16. Taira, N., K. Satoh, T. Yanagisawa, and Y. Imai. 1979. Pharmacological profile of a new coronary vasodilator drug 2-nicotinamideoethyl nitrate (SG-75). Clinical Exp. Pharmacol. Physiol. 6:301-316
17. Lamping, K. A., and G. J. Gross. 1985. Improved recovery of myocardial segment function following a short coronary vascular occlusion in dogs by nicorandil, a potential new anti-anginal agent, and nifedipine. J. Cardiovasc. Pharmacol. 7:158-166.

18. Yamada, T., K. Shimizu, T. Kuroiwa, and T. Ohta. 1986. Pharmacological study on the effects of nicorandil on smooth muscle of the cerebral artery. Congr. Jap. Soc. Cerebral Stroke, Tokyo.

19. Kassel, N. F., and C. G. Drake. 1983. Review of the management of saccular aneurysms. Neurol. Clinics N. Amer. 1:73-86.

20. Sasaki, T., N. F. Kassel, M. Yamashita, S. Fujiwara, and M. Zuccarello. 1982. Barrier disruption in the major cerebral arteries following experimental subarachnoid hemorrhage. J. Neurosurg. 63:433-440. 